\title{
Negoziabilità del profitto e design organizzativo: una proposta interpretativa
}

\author{
LOREDANA VOLPE ${ }^{* *}$ GIANLUCA VAGNANI
}

\begin{abstract}
Obiettivo del paper: Proporre un modello teorico-concettuale in grado di giustificare teoricamente la qualificazione del profitto come rendita negoziabile ex ante tra proprietà e top management e di chiarirne le implicazioni per il design organizzativo.

Metodologia: Revisione ed integrazione di più framework teorici: la letteratura inerente la negoziazione ex ante sulla rendita; $i$ contributi sul design organizzativo.

Risultati: Secondo il modello proposto, le organizzazioni in cui il profitto è trattato come rendita negoziabile ex ante tra proprietà e top management tendono ad essere caratterizzate da bassa formalizzazione e moderati livelli di centralizzazione e specializzazione, ossia da strutture organizzative che approssimano il modello quasi-organicistico.

Limiti della ricerca: Lo studio avanza proposizioni teorico-concettuali, non corredate da validazione empirica.

Implicazioni pratiche: La prima implicazione concerne l'eventualità che taluni stakeholder, sia interni sia esterni all'impresa, negoziando ex ante sulla propria rendita, possano influenzare l'ammontare del valore totale appropriato dall'impresa. Un secondo profilo di riflessione emerge con riguardo all'eventualità che la quasi-coalizione tra proprietà e management possa propendere verso la progettazione di strutture organizzative in grado di rafforzarne il relativo potere negoziale, ancorché inappropriate alla luce delle condizioni di contesto e delle probabilità di sopravvivenza dell'impresa. Più in generale, il modello proposto stimola la riflessione sui rapporti tra negoziabilità del profitto, strutture organizzative e relativa idoneità a favorire lo sviluppo di nuove capacità, nonché a ricomporre il conflitto di interessi tra proprietà e management.

Originalità del paper: Lo studio si situa all'intersezione tra diverse prospettive teoriche, invertendo il nesso di causalità tra profitto e design organizzativo e arricchendo il dibattito sulle fonti di eterogeneità delle imprese e dei relativi modelli organizzativi.
\end{abstract}

Parole chiave: profitto; rendita negoziabile; design organizzativo

Il presente lavoro è frutto delle riflessioni congiunte degli autori. Tuttavia si precisa che: il paragrafo 1 è da attribuirsi a Gianluca Vagnani; i paragrafi 2 e 3 sono da attribuirsi a Loredana Volpe. Il lavoro ha, inoltre, beneficiato del supporto finanziario offerto da Sapienza Università di Roma e da Regione Lazio.

** Ricercatore di Economia e Gestione delle Imprese - Sapienza Università di Roma e-mail: loredana.volpe@uniroma1.it

*** Ordinario di Economia e Gestione delle Imprese - Sapienza Università di Roma e-mail: gianluca.vagnani@uniroma1.it 
Purpose of the paper: This paper aims to develop a conceptual model, which focuses on the role of ex ante negotiation over rent between owners and executives in accounting for organizational design differences among firms.

Methodology: Development of a theoretical model based on a comprehensive review and cross-fertilization of the literature on both ex ante negotiation over rent and organizational design.

Findings: Our model posits that, when owners and executives negotiate ex ante over rent, an organizational structure tends to emerge that strengthens their bargaining power. This structure approximates the quasi-organic form, with low formalization and some degree of centralization and specialization.

Research limits: We develop detailed propositions linking the issues, but we do not provide empirical validation, which would be a logical next step.

Practical implications: A first implication concerns how different stakeholders may affect total value appropriation for the firm by negotiating ex ante over rent. A second implication refers to the attitude of the quasi-coalition between owners-managers toward building organizational structures that, although not aligned to environmental conditions and adequate to preserve firm survival, might however favor ex ante negotiation over profit. More generally, our model stimulates the debate on the relationship between profit as a negotiable rent, organizational structures and their capacity to favor the development of new capabilities and reduce owners-management conflict of interests.

Originality of the paper: This study is set at the intersection of distinct theoretical perspectives. It revers the nexus of causality between profit and organizational design thereby contributing to the debate on the sources of heterogeneity of firms and their organizational models.

Key words: profit; ex ante negotiation over rent; organizational design

\section{Introduzione}

In che misura e sotto quali condizioni è possibile ammettere che il profitto perda la sua naturale residualità, trasformandosi in rendita negoziabile ex ante? E in che modo la negoziazione ex ante su tale rendita condiziona le scelte inerenti la progettazione della struttura organizzativa (o design organizzativo) e lo sviluppo di nuove capacità?

Gli interrogativi suesposti si pongono al centro di un animato e crescente dibattito tra gli studiosi d'impresa in merito alla relazione tra vantaggio competitivo ed eterogeneità delle imprese e dei relativi modelli organizzativi (Coff, 2010, 1999; Belussi e Pilotti, 2006; Castanias e Helfat, 2001). Al proposito, il presente contributo esplora un aspetto di tale eterogeneità particolarmente significativo e saliente. Esso, infatti, avanza un modello teorico-concettuale orientato a giustificare l'ipotesi di qualificazione del profitto come rendita negoziabile ex ante tra proprietà e top management, analizzandone le implicazioni per le scelte di progettazione della struttura organizzativa. A ben guardare, il modello proposto muove da un presupposto teorico di non poco rilievo.

In presenza di date circostanze (ossia la generazione di rendite conseguenti allo sviluppo di nuove capacità organizzative) e per date categorie di portatori di 
interessi espressive di un potere negoziale (top management e proprietà), il profitto, tradizionalmente assimilato a una qualche forma di reddito economico residuale e variabile, appare risolversi in una rendita negoziabile ex ante ${ }^{1}$. A rigore dovrebbe parlarsi di quasi-rendita. L'espressione quasi-rendita è da intendersi con riferimento a grandezze predeterminabili ex ante come surplus sul costo-opportunità di soggetti partecipanti a una negoziazione, ossia tra loro interagenti al fine di conseguire reciproci obiettivi (Klein et al., 1978). La nozione di quasi-rendita rimanda, dunque, a una differenza di valore, la differenza tra il valore di una risorsa nella relazione (negoziale) per la quale essa è specifica e il valore della risorsa medesima nel miglior uso alternativo. Più specificatamente, l'assimilazione tra le categorie economiche di profitto e rendita trova un significativo antecedente in letteratura nell'ambito della cosiddetta Teoria della negoziazione ex ante sulla rendita (Rent Appropriation Theory, cfr. Coff, 2010, 2003, 1999). Tale approccio, scontando una prospettiva resource-based, ricollega, infatti, il passaggio dalla creazione di profitto alla generazione di rendite alla presenza di conoscenze e capacità «incastonate» in singoli soggetti o gruppi di soggetti e quindi all'emergere di asimmetrie di conoscenza tra distinti portatori di interessi nei confronti dell'impresa (Campbell $e t$ al., 2012; Kor e Leblebici, 2005; Hatch e Dyer, 2004; Coff, 1999). Ipotizzando che questi soggetti siano individuabili nel top management e nella proprietà, nonché ammettendo, come postulato dalla Rent Appropriation Theory, che il «capitale» (umano e finanziario) da essi apportato non possa essere totalmente controllato dall'impresa, bensì liberamente trasferito altrove in relazione a considerazioni di convenienza, ecco allora che la remunerazione spettante ai soggetti menzionati può assumere le sembianze di una quasi-rendita, ossia di quella «frazione dei proventi che eccedono l'ammontare minimo necessario per evitare che un lavoratore abbandoni un certo lavoro o un'impresa abbandoni una certa industria» (Milgrom e Roberts, 1994, p. 399). A partire da tali premesse, la teoria in esame identifica, dunque, nella negoziazione ex ante sulla rendita l'emergere di processi forieri di agevolare l'appropriazione di rendite da parte di specifici portatori di interesse nei confronti dell'impresa, i quali contribuirebbero allo sviluppo di nuove capacità

1 In merito alla distinzione tra i concetti di profitto e rendita non appare superfluo ricordare che, convenzionalmente, la letteratura economica individua distinte forme di remunerazione dei fattori della produzione in base al relativo grado di incertezza e rischio. La forma maggiormente certa, contrattualmente garantita, di remunerazione spettante a un dato soggetto rispetto a migliori alternative di impiego o allo svolgimento in proprio di un'attività di produzione economica, identifica il cosiddetto costo-opportunità. Si definisce, invece, rendita economica del soggetto il surplus, predeterminabile contrattualmente, su tale costo-opportunità. Infine, qualora condizioni di incertezza e informazione imperfetta rendano impossibile determinare contrattualmente il surplus sul costo-opportunità di un dato fattore produttivo, la remunerazione spettante a quest'ultimo prende il nome di profitto. Sul concetto di rendita, più specificatamente, appare chiarificatrice la posizione di Napoleoni, secondo il quale la rendita identifica il «reddito che il proprietario di certi beni percepisce in conseguenza del fatto che tali beni sono, o vengono resi, disponibili in quantità scarsa», cfr. Napoleoni (1956) . 
organizzative soprattutto al fine di beneficiare delle rendite economiche connesse a tale sviluppo (Coff, 2010, p. 716).

Nonostante il fascino e la coerenza delle argomentazioni sin qui esposte, le implicazioni connesse alle descritte dinamiche negoziali e appropriative per taluni rapporti e aspetti essenziali della vita e del funzionamento dell'impresa, quali le relazioni tra stakeholder-chiave (top management e proprietà) e le scelte inerenti la progettazione della struttura organizzativa, appaiono ancora poco esplorate. Obiettivo di questo studio è pertanto quello di integrare la letteratura inerente la negoziazione ex ante sulla rendita (Coff, 2010, 2003, 1999) con quella incentrata sulla progettazione della struttura organizzativa (Mintzberg, 1979; Khandwalla, 1972).

Da un lato, infatti, secondo il primo filone teorico, alcune categorie di stakeholder, facendo leva sull'esercizio del relativo potere negoziale, godrebbero di una posizione di privilegio nell'assicurarsi i ritorni economici derivanti dallo sviluppo di nuove capacità organizzative, configurandosi così un'appropriazione della rendita ex ante, ossia ancor prima che la rendita stessa sia generata o che altri riescano a realizzare le potenzialità legate allo sviluppo di nuove capacità. Dall'altro lato, scontando il presupposto che dati risultati di performance dell'impresa siano la conseguenza di date configurazioni strutturali, la letteratura di matrice organizzativa non sembra aver considerato finora il rovescio della medaglia, ossia il nesso causale tra generazione e appropriazione di rendite da parte di stakeholder-chiave dell'impresa (proprietà e top management) e tipo di struttura organizzativa prescelta.

Il modello teorico-concettuale qui proposto apre così uno spazio di discussione che si situa all'intersezione tra distinte prospettive teoriche; prospettive rivolte sia allo studio della relazione tra design organizzativo e «variabili situazionali», sia all'analisi del potere in forza del quale specifici stakeholder negoziano ex ante sulle rendite derivanti dallo sviluppo di nuove capacità. In particolare, con riguardo a quest'ultimo aspetto, sembra possibile affermare che la negoziazione ex ante sul profitto tra proprietà e top management abbia suscitato attenzione e interesse comparativamente inferiori in seno alla letteratura corrente soprattutto rispetto ad ipotesi di negoziazione ex ante sulle rendite tra stakeholder «interni» all'impresa (ad esempio tra top management e personale dipendente). Invero, secondo la letteratura di riferimento, in presenza di asimmetrie di conoscenza, la proprietà vedrebbe tipicamente ridursi il proprio potere negoziale relativamente al potere esercitabile dal top management (Coff, 2010, 1999). In parziale dissenso rispetto a questo assunto, si ritiene, invece, che la prospettiva in esame possa dirsi teoricamente fondata solo se in grado di mantenere la sua validità anche con riferimento al caso in cui proprietà e top management negoziano ex ante sulla rendita connessa allo sviluppo di nuove capacità organizzative, ancorché a scapito degli interessi di altre categorie di stakeholder.

Muovendo da tale premessa, il presente contributo sviluppa proposizioni che invertono il nesso causale tra scelte di progettazione della struttura organizzativa e processi generativi (e appropriativi) del profitto. In breve, nelle organizzazioni in cui il profitto è concepito come rendita negoziabile ex ante tra proprietà e top 
management, la progettazione della struttura organizzativa riflette il potere negoziale della coalizione tra queste categorie di stakeholder, approssimando modelli quasiorganicistici (Goffee, 1996; Goffee e Scase, 1985; Burns e Stalker, 1961).

Si ritiene che le implicazioni associate al modello proposto contribuiscano ad arricchire e a estendere la letteratura in essere sulle scelte di progettazione strutturale delle imprese, introducendo la negoziazione ex ante sul profitto tra i fattori tradizionalmente reputati in grado di spiegare l'adozione di differenti strutture organizzative lungo l'ideale continuum tra forme organicistiche e meccanicistiche (Burns e Stalker, 1961). In tal senso, lo studio della negoziazione ex ante sul profitto introduce un ulteriore fattore contingente rispetto a quelli già esplorati in letteratura tra cui, l'incertezza del contesto, (Lawrence e Lorsch, 1986; Pugh et al., 1969); il determinismo tecnologico, (Covin e Slevin, 2006; Perrow, 1967; Woodward et al., 1965); l'allineamento con la strategia d'impresa, (Chandler, 1962; Khandwalla, 1972).

\section{Quadro teorico di riferimento e sviluppo del modello concettuale}

\subsection{La teoria della negoziazione ex ante sulla rendita}

L'attenzione verso le implicazioni connesse al potere negoziale esercitato da soggetti distinti, portatori di interesse nei confronti di una comune «intrapresa», è connaturata negli studi d'impresa, sia italiani, sia d'oltreoceano. In quest'ultimo ambito, in particolare, svariati contributi dimostrano come la presenza di asimmetrie di conoscenza possa determinare opportunità di negoziazione ex ante sulla rendita in favore di talune categorie di stakeholder (Coff, 2010, p. 713). Prima di delineare più in dettaglio i contenuti del filone di studi accomunabili sotto l'etichetta di «Teoria della negoziazione ex ante sulla rendita» (Rent Appropriation Theory), non sembra superfluo evidenziare che entrambe le espressioni «stakeholder» e «asimmetrie di conoscenza» assumono, in seno ad esso, una connotazione peculiare. Da un lato, il termine stakeholder qualifica categorie di soggetti caratterizzate da un forte interesse allo sviluppo di nuove capacità, ovvero in grado di svolgere un ruolo determinante in tale sviluppo, giacché mosse dalla possibilità di catturarne la rendita economica (Coff, 2010; Teece, 1986). Dall'altro lato, la nozione di asimmetrie di conoscenza identifica condizioni di asimmetria legate non tanto al possesso di informazioni (know-what), quanto a differenze nella cosiddetta conoscenza pratica o procedurale (know-how). In particolare, secondo l'approccio in esame, nella presenza di asimmetrie di conoscenza sarebbe riscontrabile una fonte rilevante di potere negoziale. Trattasi, infatti, di asimmetrie che consentono a specifiche categorie di stakeholder di avere accesso a un patrimonio di conoscenze e capacità innovative, uniche e non egualmente accessibili ad altri stakeholder.

Più specificatamente, la Teoria della negoziazione ex ante sulla rendita ricollega la comprensione stessa del nesso tra vantaggio competitivo e generazione di profitti all'analisi delle asimmetrie di conoscenza e del potere negoziale espresso da diversi 
portatori di interessi nei confronti dell'impresa. Potere negoziale inteso, a sua volta, come meccanismo «normale» di espressione dei diritti di proprietà e, quindi, di determinazione ex ante della quota di profitto appropriabile da specifici stakeholder (Coff, 2010, p. 713). Distinguendo tra stakeholder interni (il top management) ed esterni (la proprietà), la teoria in esame enfatizza il maggior potere negoziale esercitabile dagli stakeholder interni all'impresa, specie con riferimento a imprese caratterizzate da separazione tra proprietà e controllo (Blyler e Coff, 2003; Coff e Lee, 2002; Chacar e Coff, 2000). In presenza di asimmetrie di conoscenza, tale teoria rimarca la posizione di svantaggio gravante sulla proprietà rispetto al top management. Coff e Lee (2002) dimostrano, al proposito, come, rispetto alla proprietà, il top management possa caratterizzarsi per una superiore capacità a sfruttare le asimmetrie di conoscenza, sovente attraverso forme di insider trading o più favorevoli meccanismi di incentivazione. In aggiunta, il potere negoziale della proprietà risulterebbe di per sé modesto a causa della spiccata sostituibilità delle risorse finanziarie fornite all'impresa, in particolare qualora non detenga quote tali da influenzare la valutazione del capitale espressa dal mercato (Castanias e Helfat, 1992).

In buona sostanza, può dirsi che l'approccio teorico sin qui descritto tenda a escludere l'ipotesi di negoziazione ex ante sulla rendita tra proprietà e top management, ancorché svariati studi forniscano evidenze che, viceversa, ne supportano l'ammissibilità. Diversi autori evidenziano, infatti, l'abilità della proprietà a influenzare l'appropriazione della rendita spettante ad altri stakeholder (ed all'impresa nel suo complesso). Ciò può avvenire ad esempio attraverso pratiche predatorie accomunate sotto la denominazione di tunneling (Morck e Yeung, 2004; Johnson, 2000). In aggiunta, è possibile osservare che, data la scarsità della risorsa apportata all'impresa, la proprietà può esercitare pressioni al rialzo sul prezzo del capitale e così catturare una maggior quota del «valore» totale creato (Bowman e Ambrosini, 2000). Più in generale, alla proprietà spettano tipicamente diritti residuali sul profitto, in relazione ai quali essa esercita un significativo potere negoziale (Jensen e Meckling, 1976; Smith, 1937).

Contrariamente a quanto sostenuto dalla Rent Appropriation Theory, gli studi menzionati inducono, dunque, ad assumere che proprietà e top management possano risultare direttamente coinvolti nello sviluppo di nuove capacità e quindi in grado di negoziare ex ante sulla rendita connessa a tale sviluppo. Assunzione che appare giustificabile sotto un duplice profilo.

Da un lato, è ragionevole ritenere che la proprietà, in virtù delle sue doti di imprenditorialità innovativa (Schumpeter, 1934) e di alertness (Kirzner, 1973; 1979), manifesti una significativa attitudine a riconoscere le situazioni da cui è possibile trarre un profitto investendo risorse finanziarie nello sviluppo di nuove capacità (Dierickx e Cool, 1989). In tal caso, è verosimile ritenere che la proprietà cerchi di garantirsi l'appropriazione della rendita connessa alle suddette capacità prima che il potenziale di queste ultime trovi riflesso nella valutazione complessiva dell'impresa. 
Per esemplificare, si supponga che lo sviluppo di capacità riguardi la realizzazione di un nuovo prodotto o l'attribuzione di nuove caratteristiche a un prodotto già esistente, come nel caso dell'introduzione sul mercato dell'iPod, riportato in letteratura da Coff (2010). L'idea innovativa circa la creazione di un lettore di musica digitale basato su hard disk, da sincronizzarsi tramite software con un negozio di musica digitale online, è da attribuirsi all'ingegnere americano Tony Fadell. Fadell sviluppa tale idea assai prima di incontrare Steve Jobs quale CEO della Apple Computer. Già infatti a partire dal 1995, Fadell opera presso Philips Consumer Electronics come Ingegnere capo e Responsabile delle innovazioni tecnologiche nel settore dei dispositivi portatili. È in questi anni che nasce l'idea del prodotto innovativo, in seguito denominato iPod. Tuttavia, non disponendo di risorse finanziarie e capacità complementari sufficienti allo sviluppo dell'idea (apparecchiature produttive hardware e rete di consegna dei contenuti), né riuscendo ad attrarre tali risorse attraverso la creazione di una propria impresa, Fadell avvia rapporti collaborativi con Apple Computer, finalizzati alla realizzazione del progetto iPod. La vicenda dell'iPod è esemplare per comprendere il potere negoziale della proprietà. Essa suggerisce che lo sviluppo di nuove capacità incorpora un potenziale di profitto per diversi stakeholder, la cui negoziazione ex ante è funzione del relativo potere negoziale. Nel caso riportato, gli sforzi inizialmente compiuti da un inventore per acquisire le risorse finanziarie indispensabili allo sviluppo della propria idea, producono, tuttavia, l'effetto collaterale di rivelarne il connesso potenziale di generazione di rendite ad altri stakeholder, orientati a investire nello sviluppo del nuovo prodotto e a negoziare ex ante sulla rendita derivante da tale sviluppo.

Dall'altro lato, l'attribuzione alla proprietà di un significativo potere negoziale appare giustificata dalla possibile «contrattualizzazione» della rendita che, nell'ambito del complessivo profitto creato, spetterebbe al top management per l'efficace applicazione delle proprie competenze ai processi ed alle attività d'impresa. Competenze manageriali non appropriabili dalla proprietà, né acquisibili sul mercato. In effetti, non appare del tutto infondato ritenere che, intravedendo le opportunità di rendita associate allo sviluppo di nuove capacità, il top management possa indirizzare l'impresa verso un più spinto ed efficace utilizzo delle risorse disponibili - ivi incluse quelle di natura finanziaria, apportate dalla proprietà (Golinelli, 2011).

In buona sostanza, sulla base di quanto sinora argomentato, il modello concettuale proposto in questo studio estende ed arricchisce la teoria della negoziazione ex ante sulla rendita, rivalutando l'eventualità che anche la proprietà e non solo il top management - eserciti un potere negoziale adeguato a consentirle di trasformare la propria remunerazione da quantità residuale a rendita negoziabile $e x$ ante. Il modello contempla, dunque, la possibile interazione tra stakeholder interni (top management) ed esterni all'impresa (proprietà), attraverso un complesso gioco di comportamenti, formali e informali, che assume caratteri quasi-coalizzativi. Più specificatamente, l'espressione quasi-coalizione tra proprietà e top management implica che quest'ultimo non sfrutti le asimmetrie di conoscenza di cui gode a danno degli interessi della prima, profilandosi in tal senso un peculiare contesto di 
governance (riscontrabile, ad esempio, nell'ambito dei cosiddetti family businesses), in cui la tradizionale conflittualità dei rapporti di agenzia appare fortemente ridimensionata. Alla base del formarsi della quasi-coalizione tra top management $\mathrm{e}$ proprietà è la possibilità stessa di esercitare un forte potere negoziale da parte di entrambi, ossia di agire verso un obiettivo mutualmente condiviso, identificabile nella negoziabilità ex ante della rendita connessa allo sviluppo di nuove capacità.

A ben guardare, dunque, il modello concettuale proposto appare imperniato su due assunti fortemente interdipendenti tra loro: (i) la negoziabilità ex ante del profitto; (ii) la quasi-coalizione tra proprietà e management. Per un verso, il formarsi della quasi-coalizione assume le sembianze di un meccanismo decisionale e di azione, teso al superamento di situazioni di conflitto di interessi e quindi foriero di agevolare la negoziazione ex ante sulla rendita. Per altro verso, la qualificazione del profitto come rendita negoziabile ex ante condiziona l'azione di governo dell'impresa, orientando il top management verso un impiego delle risorse disponibili (ivi incluse le proprie competenze e le risorse finanziarie apportate dalla proprietà), funzionale allo sviluppo di nuove capacità, con il connesso potenziale di generazione di rendite.

\subsection{Profitto come rendita negoziabile ex ante e design organizzativo: una proposta di inquadramento concettuale}

Il tema della negoziazione ex ante sul profitto tra proprietà e top management, quale determinante delle scelte relative alla progettazione della struttura organizzativa dell'impresa (organizational design), appare ancora poco esplorato, specie nell'ambito della letteratura di matrice organizzativa. Le questioni affrontate dagli studiosi di design organizzativo risultano varie e variegate, polarizzandosi su due fronti di ricerca (Astley e van de Ven, 1983). Il primo fronte si focalizza sulla relazione tra dimensioni della struttura organizzativa e variabili situazionali, tra cui rientrano fattori contingenti relativi al contesto in cui opera l'impresa (Khandwalla, 1977; Lawrence e Lorsch, 1967; Woodward et al., 1965 ) o la dimensione di questa (Blau, 1970; Pugh et al., 1969). Viceversa, il secondo fronte di ricerca sottopone a critica la precedente prospettiva strutturalista, in quanto tendente ad attribuire al top management un ruolo meramente reattivo rispetto ai cambiamenti del contesto (Child, 1972). Al contrario, secondo gli autori che si riconoscono in questa seconda prospettiva, le strutture organizzative risulterebbero continuamente create, plasmate e modificate dal top management in maniera proattiva (Astley e van de Ven, 1983, p. 253). Tali autori, dunque, attribuiscono al top management considerevoli gradi di libertà sia nel riconoscere la natura sistemica delle scelte inerenti la progettazione della struttura organizzativa (Rivkin e Siggelkow, 2003), sia nell'identificare gli elementi specifici da includere nella progettazione (Siggelkow, 2002).

Ai fini del presente contributo un elemento della corrente teorica in esame appare particolarmente significativo, ossia l'identificazione del profitto come driver fondamentale delle scelte di governo. Nota al proposito Child (1972, p. 11): «亡 consuetudine assumere che un certo livello [di profitto] rappresenti il ritorno ritenuto 
almeno sufficiente ad assicurare la disponibilità delle risorse necessarie all'implementazione dei piani di sviluppo presenti e futuri».

In particolare, le posizioni espresse dagli autori ora menzionati possono dirsi di rilievo per lo studio svolto, in quanto forniscono taluni presupposti teorici funzionali all'elaborazione del modello concettuale qui proposto. Presupposti che affondano le proprie radici in un complesso di contributi, orientati a considerare la struttura organizzativa quale risultante di veri e propri processi di negoziazione tra la «coalizione dominante» (cfr. Cyert e March, 1963) e molteplici attori interni ed esterni all'impresa (Pfeffer e Salancik, 1978, 1977). Al proposito, alcuni autori (cfr. Astley e Zajac, 1991) argomentano la necessità di considerare congiuntamente le questioni concernenti il design organizzativo e quelle relative all'esercizio di un potere negoziale. Ad esempio, a livello di singole unità organizzative, l'esercizio di potere negoziale appare sovente risolversi in condizioni di «accoppiamento stretto» (tight coupling), che preludono alla configurazione di modelli meccanicistici di struttura organizzativa (Burns e Stalker, 1961).

Ammettendo, dunque, che il design organizzativo possa essere il riflesso di dinamiche coalizionali, il ragionamento sin qui svolto porta a ritenere che, nelle suddette circostanze, la progettazione della struttura organizzativa bilanci la generazione di profitto per l'impresa rispetto alla quota, di esso, appropriabile ex ante dalla coalizione tra proprietà e top management. In altri termini, agendo sulle scelte di progettazione della struttura organizzativa, il formarsi della quasicoalizione tra proprietà e top management inciderebbe sull'entità del profitto negoziabile (e quindi appropriabile) ex ante dalla coalizione medesima.

Tale argomentazione trova parziale riscontro nella letteratura comportamentista, sebbene riferita agli effetti del potere negoziale singolarmente esercitato dal management o dalla proprietà sulle rendite spettanti ad altri stakeholder o sul profitto totale d'impresa. Di recente, ad esempio, David et al., (2010) forniscono evidenze empiriche che attribuiscono alla proprietà significative capacità a limitare le rendite disponibili per altri stakeholder, specie in rapporto a date traiettorie di sviluppo dell'impresa, quali la diversificazione. Anche Coff (2010, p. 712) segue una linea di pensiero analoga a quella ora esposta con riferimento alla scelta della forma organizzativa da parte del team di fondatori dell'impresa, osservando che «ad esempio, è possibile che un imprenditore scelga quella forma organizzativa che pur non massimizzando il valore totale creato, riesce tuttavia ad incrementare la porzione che esso sarà in grado di catturare». Donaldson e Davis (1991), infine, esponenti della cosiddetta Stewardship Theory, ammettono esplicitamente la possibilità che il top management cerchi di massimizzare le performance organizzative o il profitto per la proprietà nella misura in cui ciò possa risultare funzionale a preservare l'integrità della coalizione tra management e proprietà.

In effetti, sostenere che top management e proprietà possano ridurre il profitto a rendita negoziabile ex ante non implica necessariamente che essi si trovino in posizione più favorevole, rispetto ad altri stakeholder, al momento dell'appropriazione del profitto medesimo. Di conseguenza, al fine di garantirsi l'appropriazione del profitto, la quasi-coalizione tra proprietà e top management 
tenderà a plasmare la struttura organizzativa secondo configurazioni tali da rafforzare il proprio potere negoziale, relativamente ad altri portatori di interesse.

Da tutto quanto sin qui argomentato discende la prima proposizione a base del modello teorico-concettuale proposto:

P1: Nelle organizzazioni in cui proprietà e top management trattano il profitto come rendita negoziabile ex ante, la progettazione della struttura organizzativa è da intendersi quale risultante del potere negoziale della quasi-coalizione tra le suddette categorie di stakeholder.

Al fine di una più approfondita comprensione delle implicazioni derivanti dalla negoziazione ex ante sul profitto per le scelte di progettazione della struttura organizzativa, non appare superfluo ricordare che, in generale, la struttura di un'organizzazione è tipicamente definita «dalla somma totale dei modi in cui essa divide il lavoro tra compiti distinti e realizza, poi, il coordinamento tra gli stessi» (Mintzberg, 1979, p. 2). L'analisi delle dimensioni della struttura organizzativa e la giustificazione dell'eterogeneità che, sotto questo profilo, caratterizza le organizzazioni hanno impegnato a lungo la riflessione degli studiosi (Lawrence e Lorsch, 1967; Woodward et al., 1965; Burns e Stalker, 1961). In particolare, uno dei più noti e accreditati inquadramenti teorici distingue tra strutture meccanicistiche e organicistiche (Burns e Stalker, 1961).

Una struttura di tipo meccanicistico si caratterizza per la presenza di linee gerarchiche verticali, numerosi dipartimenti, scarsa decentralizzazione decisionale e molteplici regole e procedure. L'autorità è centralizzata al vertice della struttura, standardizzazione e formalizzazione dei ruoli risultano elevate, i compiti individuali sono specificati rigidamente e le interazioni seguono una direzione verticale. Dall'altro lato, una struttura di tipo organicistico si distingue per la sua tendenziale apertura, il minor numero di regole, l'estesa decentralizzazione, la meno rigida definizione di metodi, compiti e poteri e la capacità di indurre interazioni orizzontali (Marsden et al., 1994; Bantel, 1993).

I primi studi sulle dimensioni della struttura organizzativa risalgono agli anni ' 40 dello scorso secolo. In seguito, soprattutto a partire dal 1970, l'attenzione degli studiosi è andata spostandosi verso l'identificazione di svariate tipologie strutturali in relazione al grado in cui esse risultano caratterizzate da tre distinte dimensioni: formalizzazione, centralizzazione e specializzazione. L'articolato combinarsi di queste dimensioni determina ciò che la letteratura qualifica come «grado di organicità», corrispondente alla posizione astrattamente occupata da una struttura organizzativa lungo l'ideale continuum tra strutture meccanicistiche ed organicistiche.

Alla luce del concetto di grado di organicità e sviluppando ulteriormente il ragionamento seguito nel formulare la proposizione $\mathrm{P} 1$, sembra possibile argomentare che, laddove la struttura organizzativa rappresenti la risultante della capacità, espressa dalla coalizione tra proprietà e management, di negoziare ex ante sulla rendita, il grado di ciascuna dimensione strutturale (formalizzazione, 
centralizzazione e specializzazione) debba riflettere tale effetto. Detto altrimenti, nelle imprese in cui proprietà e top management pongono in essere comportamenti quasi-coalizzativi finalizzati a negoziare ex ante sul profitto, il grado di organicità della struttura organizzativa sarà plasmato in funzione delle istanze di appropriazione di profitto, espresse dalla quasi-coalizione stessa. Ecco allora profilarsi configurazioni strutturali che consentono al top management di mantenere un certo livello di autonomia e discrezionalità, ancorché assicurando alla proprietà di salvaguardare l'esercizio delle proprie prerogative di controllo. Configurazioni che approssimano, a ben vedere, la tipologia strutturale codificata in letteratura come quasi-organicistica (Goffee e Scase, 1985).

La denominazione quasi-organicistica è da ricondursi alla capacità di queste strutture ad accomodare potenziali tensioni tra proprietà e top management, legate alla presenza di asimmetrie di conoscenza. In un pionieristico contributo, Goffee e Scase (1985) evidenziano come (soprattutto in imprese a controllo familiare) il management scelga deliberatamente di configurare la struttura organizzativa secondo il modello quasi-organicistico, giacché quest'ultimo ne rafforza le capacità di controllo.

Più specificatamente, con riguardo alle principali dimensioni strutturali (formalizzazione, centralizzazione e specializzazione), l'ipotesi di negoziazione ex ante sul profitto tra proprietà e top management appare suscettibile di condurre a strutture quasi-organicistiche contraddistinte da bassa formalizzazione e moderati livelli di centralizzazione e specializzazione. La combinazione delle tre dimensioni strutturali nel senso ora esposto è suggerita dalla comprovata attitudine delle strutture quasi-organicistiche a risolvere due esigenze contrapposte, emergenti anche nell'ipotesi di negoziazione ex ante sul profitto (Goffee, 1996; Goffee e Scase 1985).

Per un verso, queste strutture tendono a caratterizzarsi per limitati livelli di centralizzazione e scarsa formalizzazione, ciò che consente al top management di mantenere sufficiente discrezionalità decisionale. Per altro verso, il rischio che il top management possa privare la proprietà del profitto ad essa spettante appare ridursi nelle strutture quasi-organicistiche, grazie alla bassa formalizzazione del processo decisionale, che permette alla proprietà di far salva «l'abilità a controllare e orientare l'azione organizzativa» (Pfeffer e Salancik, 1978, p. 27). In altri termini, attraverso la creazione di strutture quasi-organicistiche, l'autorità decisionale del management risulta limitata all'assunzione solo di alcune decisioni, in alcuni momenti specifici, nell'ambito di un modello non-gerarchico di divisione del lavoro (Goffee e Scase, 1985, p. 58).

Infine, in termini di specializzazione (ovvero di divisione di compiti e attività tra posizioni), le strutture quasi-organicistiche non manifestano l'elevata specializzazione che tipicamente distingue il modello organicistico puro in relazione ad una maggiore capacità di adattamento all'ambiente (Chakravarthy, 1982; Mintzberg, 1979; Kast e Rosenzweig, 1973). Viceversa, queste strutture tendono a presentare livelli moderati di specializzazione, il che ne riconferma la corrispondenza all'ipotesi di negoziazione ex ante sul profitto. La scarsa 
specializzazione, infatti, produce svariati effetti funzionali a tale ipotesi, i quali si esplicano, in buona sostanza, nella maggiore controllabilità dei comportamenti individuali e nella possibilità di rafforzare il controllo formale su numerose attività.

Alla luce delle argomentazioni sopra esposte, si giunge a formulare la seconda proposizione fondante il modello teorico-concettuale avanzato.

P2: Le organizzazioni, in cui proprietà e top management trattano il profitto come rendita negoziabile ex ante, tendono ad essere caratterizzate da bassa formalizzazione e moderati livelli di centralizzazione e specializzazione, ossia da strutture organizzative che approssimano il modello quasi-organicistico.

\section{Implicazioni del modello teorico-concettuale proposto: alcune riflessioni}

Integrando la letteratura inerente la qualificazione del profitto come rendita negoziabile ex ante con gli approcci di matrice organizzativa concernenti le scelte di design organizzativo, il presente studio sviluppa un modello-concettuale in grado di evidenziare le implicazioni della negoziazione ex ante sul profitto tra proprietà e top management per le scelte di progettazione della struttura organizzativa e lo sviluppo di nuove capacità. Si ritiene che al modello proposto si associno molteplici e significative implicazioni, sia per la teoria d'impresa, sia sotto il profilo della prassi manageriale.

\subsection{Implicazioni per la teoria d'impresa}

Al presente studio si associano svariate implicazioni per la teoria d'impresa. In primo luogo, esso estende e arricchisce la letteratura sui processi generativi ed appropriativi del profitto, enfatizzando il potere negoziale della quasi-coalizione tra proprietà e top management. In particolare, le proposizioni a fondamento del modello concettuale elaborato contribuiscono allo sviluppo di una prospettiva nuova, che appare idonea a fornire una spiegazione, teoricamente fondata, alle fonti di eterogeneità delle imprese, dei connessi risultati in termini di profitto e modelli organizzativi (Coff, 2010, p. 712). A ben guardare, l'analisi svolta istituisce una peculiare e plausibile relazione tra sviluppo di nuove capacità organizzative, quale determinante di situazioni di vantaggio competitivo, e potere negoziale espresso dalla quasi-coalizione tra proprietà e top management.

In secondo luogo, il lavoro discute le implicazioni della negoziazione ex ante sul profitto per le scelte di design organizzativo. Pur riconoscendo il profondo e rilevante impatto che la turbolenza e la complessità del contesto esercitano sulle strutture organizzative, il modello teorico qui proposto reinterpreta la tradizionale letteratura di matrice organizzativa. Dati i fattori contingenti di contesto, la progettazione della struttura organizzativa sembra, infatti, delinearsi quale esito di determinanti distinte da quelle tipicamente individuate dagli studiosi di design 
organizzativo, tra cui la natura della tecnologia (Woodward et al., 1965), le interdipendenze tra unità organizzative (Thompson, 1967), il cambiamento del contesto (Lawrence e Lorsch, 1967; Burns e Stalker, 1961), le esigenze informative (Galbraith, 1973) o, più di recente, il potere dei capi dipartimentali (Siggelkow e Rivkin, 2005).

\subsection{Implicazioni per l'azione di governo dell'impresa e lo sviluppo di nuove capacità}

Dal punto di vista della prassi manageriale, è possibile evidenziare un duplice livello di implicazioni associate al modello proposto.

Il primo livello concerne la necessità di ricerca futura sul modo in cui specifiche categorie di stakeholder, agendo in vista di garantirsi l'appropriazione di rendite economiche (la «fetta della torta»), possano influenzare, in ultima istanza, il valore totale appropriato dall'impresa («la dimensione della torta»). Sotto questo profilo, emerge soprattutto la criticità dei peculiari schemi di accordo negoziale tra proprietà e top management, con i connessi meccanismi di incentivazione per quest'ultimo. Con riguardo ai primi, appare ragionevole ipotizzare che un possibile accordo negoziale tra proprietà e top management consista semplicemente nel determinare ex ante il saggio di rendimento che la proprietà sarà in grado di appropriare in seguito allo sviluppo di nuove capacità organizzative e data la remunerazione spettante al top management. In tal caso, occorre tuttavia osservare che, soprattutto in presenza di cospicue asimmetrie di conoscenza in favore del top management, l'impresa (e la sua proprietà) potrebbero ritrovarsi esposte a un duplice rischio. Da un lato, il top management potrebbe esercitare pressioni sulla proprietà finalizzate ad aprire opportunità di ri-negoziazione ex post della propria quasi-rendita. Fattispecie plausibile, in particolare, qualora il «capitale umano» del top management abbia carattere fortemente firm-specific (Wang et al., 2009; Peteraf, 1993). Dall'altro lato, emerge un più generale rischio di hold up conseguente all'eventualità che il top management, insoddisfatto della propria remunerazione, possa decidere di abbandonare l'impresa (e dissolvere conseguentemente la coalizione con la proprietà) (cfr. eg. Wright et al., 2013). Si ritiene, pertanto, imprescindibile che futuri sforzi di ricerca siano diretti anche ad approfondire lo studio dei sistemi di incentivazione più coerenti con l'ipotesi di negoziabilità ex ante del profitto tra proprietà e top management. In particolare, significativi avanzamenti dell'analisi potrebbero derivare da una migliore comprensione dei «meccanismi di isolamento» capaci di trattenere il top management dal portare altrove le proprie competenze e il proprio patrimonio di conoscenza. Ad esempio, è possibile ipotizzare che, oltre alla porzione di rendita negoziata ex ante con la proprietà o indipendentemente da questa, al top management spettino benefici non pecuniari (quali l'accesso a reti di relazioni sociali rilevanti, un ambiente di lavoro stimolante e non facilmente imitabile da altre imprese, una cultura d'impresa ispirata a valori di sostenibilità, promozione della persona e di condotte socialmente responsabili). Benefici capaci di creare condizioni idiosincratiche, che non solo concorrono a «immobilizzare» il top 
management, ma ne stimolano ulteriormente la propensione ad agire nell'interesse della coalizione con la proprietà. Più in generale, dal punto di vista dei rapporti di agenzia, il presente studio appare suggerire un'attenuazione del conflitto di interessi tra proprietà e management, introducendo la negoziazione ex ante sul profitto quale significativo deterrente ai tradizionali meccanismi di incentivazione e allineamento di interessi, postulati dalla Teoria dell'Agenzia.

In aggiunta, un secondo livello di implicazioni emerge dall'assegnare alla quasicoalizione tra proprietà e top management significative capacità ad orientare le scelte di progettazione verso strutture organizzative che, pur potendo apparire inappropriate alla luce delle condizioni di contesto e di sopravvivenza dell'impresa, consentono di incrementarne il potere negoziale, con le relative implicazioni in termini di appropriazione ex ante della relativa rendita. Questa nuova ottica apre interrogativi di rilievo: fino a che punto la progettazione di una struttura organizzativa che favorisce l'appropriazione del profitto può dirsi ottima o quantomeno soddisfacente dal punto di vista della possibilità di realizzare nuove combinazioni produttive e stimolare, quindi, l'innovazione? Entro quali limiti e in quali circostanze essa può considerarsi sostenibile? Tali interrogativi offrono un terreno fertile sul quale la futura ricerca, teorica ed empirica, potrà utilmente svolgersi.

A fronte del contributo apportato a teoria e prassi, il modello concettuale sviluppato appare suscettibile di proficui sviluppi. In particolare, accanto alla necessità di sottoporre a verifica empirica le proposizioni avanzate, l'analisi delle implicazioni connesse alla negoziazione ex ante sulla rendita, con riferimento all'intera struttura organizzativa dell'impresa, può senz'altro beneficiare di ulteriori approfondimenti. È noto, infatti, come una consolidata letteratura organizzativa ricolleghi la soluzione di istanze di specializzazione e coordinamento dell'attività economica alla possibilità di progettare strutture organizzative caratterizzate dalla compresenza di criteri tecnici ed istituzionali (cfr. ad esempio Zucker, 1987; Perrow, 1985, 1970; Scott e Meyer, 1983; Hirsch, 1975). I primi sarebbero funzionali ad assicurare lo svolgimento in sé del processo produttivo; i criteri istituzionali garantirebbero, invece, la conformità della struttura organizzativa rispetto ad attese sociali e culturali. Aderendo a tale impostazione, non è possibile escludere che gli effetti della negoziazione ex ante sul profitto tra top management e proprietà, con le connesse questioni appropriative, possano prodursi esclusivamente sul versante istituzionale del funzionamento della singola organizzazione. In tal caso, emerge un'evidente limitazione alla generalizzabilità delle conclusioni raggiunte in questo studio, le quali riguarderebbero, dunque, un singolo aspetto delle scelte di progettazione organizzativa. In particolare, verificandosi l'ipotesi in esame, eventuali implicazioni negative per la sopravvivenza dell'impresa, nascenti da situazioni di disparità nell'esercizio del potere negoziale tra stakeholder, non interesserebbero il versante tecnico, da cui dipende, a monte, la possibilità stessa che un profitto sia generato. Si ritiene, pertanto, che l'estensione dell'analisi nel senso ora esposto possa fornire rilevanti opportunità di affinamento del modello sviluppato. 
A ben guardare, tuttavia, i limiti sin qui evidenziati rafforzano l'auspicio da parte di chi scrive che altri studiosi accettino la sfida di approfondire teoricamente e sottoporre a verifica empirica le proposizioni avanzate in questo contributo.

\section{Bibliografia}

ALVAREZ S.A. (2007), "Entrepreneurial rents and the theory of the firm", Journal of Business Venturing, vol. 22, n. 3, pp. 427-442.

ASTLEY W.G., VAN DE VEN A.H. (1983), "Central perspectives and debates in organization theory", Administrative Science Quarterly, vol. 28, n. 2, pp. 245-273.

ASTLEY W.G., ZAJAC E.J. (1991), "Intraorganizational power and organizational design: reconciling rational and coalitional models of organization", Organization Science, vol. 2 , n. 4 , pp. 399-411.

BANTEL K.A. (1993), "Top team, environment, and performance effects on strategic planning formality", Group \& Organization Management, vol. 18, n. 4, pp. 436-458.

BELUSSI F., PILOTTI L. (2006), "Eterogeneità delle imprese e varietà dei modelli organizzativi", in Cainelli G., De Liso N. (a cura di), Organizzazioni, conoscenze e sistemi locali, Franco Angeli, Milano.

BLAU P.M. (1970), "A formal theory of differentiation in organizations", American Sociological Review, vol. 35, n. 2, pp. 201-218.

BLYLER M., COFF R.W. (2003), "Dynamic capabilities, social capital, and rent appropriation: ties that split pies", Strategic Management Journal, vol. 24, n. 7, pp. 677-686.

BOWMAN C., AMBROSINI V. (2000), "Value creation versus value capture: towards a coherent definition of value in strategy", British Journal of Management, vol. 11, n. 1, pp. 115.

BURNS T., STALKER G.M. (1961), The management of innovation, University of Illinois at Urbana-Champaign's Academy for Entrepreneurial Leadership, Historical Research Reference in Entrepreneurship.

CAMPBELL B.A., COFF R., KRYSCYNSKI D. (2012). "Rethinking sustained competitive advantage from human capital", Academy of Management Review, vol. 37, pp. 376395.

CASTANIAS R.P., HELFAT C.E. (2001), "The managerial rents model: Theory and empirical analysis", Journal of Management, vol. 27, n. 6, pp. 661-678.

CHACAR A., COFF R. (2000), The knowledge-based employees paradox. Winning Strategies in a Deconstructing World, John Wiley \& Sons Ltd, London.

CHAKRAVARTHY B.S. (1982), "Adaptation: A promising metaphor for strategic management", Academy of Management Review, vol. 7, n. 1, pp. 35-44.

CHANDLER A.D. (1962), Strategy and structure: Chapters in the history of the American enterprise, Massachusetts Institute of Technology, Cambridge.

CHILD J. (1972), "Organizational structure, environment and performance: The role of strategic choice", Sociology, vol. 6, n. 1, pp. 1-22.

COFF R.W. (1999), "When competitive advantage doesn't lead to performance: The resource-based view and stakeholder bargaining power", Organization Science, vol. 10, n. 2, pp. 119-133. 
COFF R.W. (2003), "Bidding wars over R\&D-intensive firms: Knowledge, opportunism, and the market for corporate control", Academy of Management Journal, vol. 46, n. 1, pp. 74-85.

COFF R.W. (2010), "The coevolution of rent appropriation and capability development", Strategic Management Journal, vol. 31, n. 7, pp. 711-733.

COFF R.W., LEE P.M. (2002), "Insider trading as a vehicle to appropriate rent from R\&D", Strategic Management Journal, vol. 24, n. 2, pp. 183-190.

COVIN J.G., SLEVIN D.P. (2006), "Strategic management of small firms in hostile and benign environments", Strategic Management Journal, vol. 10, n. 1, pp. 75-87.

CYERT R.M., MARCH J.G. (1963), A behavioral theory of the firm, Prentice Hall, Englewood Cliffs.

DAVID P., O'BRIEN J.P., YOSHIKAWA T., DELIOS A. (2010), "Do shareholders or stakeholders appropriate the rents from corporate diversification? The influence of ownership structure", Academy of Management Journal, vol. 53, n. 3, pp. 636-654

DIERICKX I., COOL K. (1989), “Asset stock accumulation and sustainability of competitive advantage", Management Science, vol. 35, n. 12, pp. 1504-1511.

DONALDSON L., DAVIS J.H. (1991), "Stewardship theory or agency theory: CEO governance and shareholder returns", Australian Journal of Management, vol. 16, $\mathrm{n}$. 1, pp. 49-64.

GALBRAITH JR. (1973), Designing complex organizations, Addison-Wesley Longman Publishing Co., Inc., Boston.

GATTI M., BIFERALI D., VOLPE L. (2009), "Il governo dell'impresa tra profitto e creazione di valore", Sinergie, n. 79, pp. 145-179.

GOFFEE R. (1996), "Understanding family businesses: issues for further research", International Journal of Entrepreneurial Behaviour \& Research, vol. 2, n. 1, pp. 3648.

GOFFEE R., SCASE R. (1985), "Proprietorial control in family firms: Some functions of 'quasi-organic' management systems", Journal of Management Studies, vol. 22, n. 1, pp. 53-68.

GOLINELLI G.M. (2011), L’Approccio Sistemico Vitale (ASV) al Governo dell'Impresa, Cedam, Padova.

GOLINELLI G.M., PROIETTI L., VAGNANI G. (2008), "L'azione di governo tra competitività e consonanza", in GOLINELLI G.M. (2008), L'Approccio Sistemico al Governo dell'impresa. Verso la Scientificazione dell'Azione di Governo, Cedam, Padova.

GOLINELLI G.M., VOLPE L. (2012), Consonanza, Valore, Sostenibilità. Verso l'Impresa Sostenibile, Cedam, Padova.

HATCH N.W., DYER J.H. (2004), "Human capital and learning as a source of sustainable competitive advantage", Strategic Management Journal, vol. 25, pp. 1155-1178.

HIRSCH P.M. (1975), "Organizational effectiveness and the institutional environment", Administrative Science Quarterly, vol. 20, n. 3, pp. 327-344.

JENSEN M.C., MECKLING W.H. (1976), "Theory of the firm: Managerial behavior, agency costs and ownership structure", Journal of Financial Economics, vol. 3, n. 4, pp. 305360.

JOHNSON S. (2000), “Tunneling”, American Economic Review, vol. 90, n. 2, pp. 22-27.

KAST F.E., ROSENZWEIG J.E. (1973), Contingency views of organization and management, Sra, Chicago.

KHANDWALLA P.N. (1972), "Environment and its impact on the organization", International Studies of Management \& Organization, vol. 2, n. 3, pp. 297-313. 
KHANDWALLA P.N. (1977), The design of organizations, Harcourt Brace Jovanovich, New York.

KIRZNER I.M. (1973), Competition and Entrepreneurship, University of Chicago Press, Chicago.

KIRZNER I.M. (1979), Perception, Opportunity and Profit: Studies in the Theory of Entrepreneurship, University of Chicago Press, Chicago.

KLEIN B., CRAWFORD R.G., ALCHIAN A.A. (1978), "Vertical integration, appropriable rents, and the competitive contracting process", Journal of Law and Economics, vol. 21, n. 2, pp. 297-326.

KOR Y.Y., LEBLEBICI H. (2005), "How do interdependencies among human-capital deployment, development, and diversification strategies affect firms' financial performance? ", Strategic Management Journal, vol. 26, pp. 967-985.

LAWRENCE P.R., LORSCH J.W. (1967), "Differentiation and integration in complex organizations", Administrative Science Quarterly, vol. 12, n. 1, pp. 1-47.

LAWRENCE P.R., LORSCH J.W. (1986), Organization and Environment: Managing Differentiation and Integration, Harvard Business School Classics.

MARSDEN P.V., COOK C.R., KALLEBERG A.L. (1994), "Organizational Structures Coordination and Control”, American Behavioral Scientist, vol. 37, n. 7, pp. 911-929.

MILGROM P., ROBERTS J. (1992), Economics, organization and management, Prentice Hall, Englewood Cliffs, (ed. italiana, Economia, organizzazione e management, Il Mulino, Bologna, 1994).

MINTZBERG H. (1979), The structuring of organizations: A synthesis of the research, University of Illinois at Urbana-Champaign's Academy for Entrepreneurial Leadership Historical Research Reference in Entrepreneurship.

MORAN P., SIMONI M., VAGNANI G. (2011), "Becoming the best: by beating or ignoring the best? Toward an expanded view of the role of managerial selection in complex and turbulent environments", Journal of Management and Governance, vol. 15, n. 3, pp. 447-481.

MORCK R., YEUNG B. (2004), "Family control and the rent-seeking society", Entrepreneurship Theory and Practice vol. 28, n. 4, pp. 391-409.

NAPOLEONI C. (1956), Dizionario di economia politica, Edizioni di Comunità, Torino.

PERROW C. (1967), "A framework for the comparative analysis of organizations", American Sociological Review, vol. 32, n. 2, pp. 194-208.

PERROW C. (1970), Organizational analysis: a sociological view, Wadsworth, Belmont.

PERROW C. (1985), "Review essay: Overboard with mith and symbols", American Journal of Sociology, vol. 91, n.1, pp. 151-155.

PETERAF M.A. (1993), "The cornerstones of competitive advantage: a resource-based view", Strategic Management Journal, vol. 14, n. 3, pp. 179-191.

PFEFFER J., SALANCIK G.R. (1977), "Organization design: The case for a coalitional model of organizations", Organizational Dynamics, vol. 6, n. 2, pp. 15-29.

PFEFFER J., SALANCIK G.R. (1978), The external control of organisations, Stanford Business Classics, Stanford.

PUGH D.S., HICKSON D.J., HININGS C.R., TURNER C. (1969), "The context of organization structures", Administrative Science Quarterly, vol. 14, n. 1, pp. 91-114.

RIVKIN J.W., SIGGELKOW N. (2003), "Balancing search and stability: Interdependencies among elements of organizational design", Management Science, vol. 49, n. 3, pp. 290-311. 
SCOTT W.R., MEYER J.W. (1983), "The organization of societal sectors", in Meyer J.W., Scott W.R. (a cura di), Organizational Environments: Ritual and Rationality, Sage, New York, pp. 129-153.

SCHUMPETER J.A. (1934), The theory of economic development, Harvard University Press, Cambridge (MA).

SIGGELKOW N. (2002), "Evolution toward fit", Administrative Science Quarterly, vol. 47, n. 1 , pp. 125-159.

SIGGELKOW N., RIVKIN J.W. (2005), "Speed and search: Designing organizations for turbulence and complexity", Organization Science, vol. 16, n. 2, pp. 101-122.

SMITH A. (1937), The Wealth of Nations (1776), Modern Library, New York.

TEECE D.J. (1986), "Profiting from technological innovation: Implications for integration, collaboration, licensing and public policy", Research Policy, vol. 15, n. 6, pp. 285305.

THOMPSON J.D. (1967), Organizations in action, McGraw-Hill, New York.

VAGNANI G., VOLPE L. (2009), "Struttura formale, informale e prestazioni individuali", Sviluppo \& Organizzazione, vol. 234, pp. 18-36.

WANG H.C., HE J., MAHONEY J. (2009), "Firm-specific knowledge resources and competitive advantage: The roles of economic- and relationship-based employee governance mechanisms", Strategic Management Journal, vol. 30, n. 12, pp. 12651285.

WOODWARD J., DAWSON S., WEDDERBURN D. (1965), Industrial organization: Theory and practice, Oxford University Press, London.

WRIGHT P.M., COFF R., MOLITERNO T.P. (2013), "Strategic Human Capital Crossing the Great Divide", Journal of Management, in corso di pubblicazione, doi: 0149206313518437.

ZUCKER L.G. (1987), "Institutional theories of organization”, Annual Review of Sociology, vol. 13 , n. 1, pp. 443-464. 